\title{
Contribución al conocimiento de la distribución de quirópteros en el norte y centro de Portugal
}

\author{
PAulo Barros 1

\begin{abstract}
${ }^{1}$ Laboratorio de Ecologia Aplicada, CITAB — Centro de Investigação e Tecnologias Agroambientais e Biológica. Universidade de Trás-
\end{abstract} \\ os-Montes e Alto Douro, 5000-911 Vila Real, Portugal. \\ Correo electrónico del autor: pbarros@utad.pt
}

DOI: http://dx.doi.org/10.14709/BarbJ.5.1.2012.04

English title: Contribution to the knowledge of bat distribution in northern and central Portugal

\begin{abstract}
In all, 25 species of bats are known from mainland Portugal, all of which are protected by law (Bern Convention, Habitats Directive, Bonn Convention and the Agreement on the Conservation of Populations of European Bats (EUROBATS); likewise, their roosts are also protected by the Bern Convention and the European Habitats Directive. In recent decades a worldwide decline in bat populations has occurred and Portugal, where nine species are listed as endangered (Critically endangered CR, Endangered EN or Vulnerable VU according to IUCN categories), is no exception. The data presented in this paper are the result of fieldwork conducted in 2009-2011 in northern and central Portugal and provide 368 new records from 53 trapping sessions. The data given for 22 bat species expands their distribution and provides new information on ecology and biology for most of the listed species. Ninety of the 368 records are new for the 10x10 km squares.
\end{abstract}

Keywords: Bats, Sites of Community Importance, distribution, Iberian Peninsula, Portugal.

Resumen: En el Portugal continental están presentes 25 especies de quirópteros. Todas las especies están protegidas por ley (Convención de Berna, Directiva Hábitats, Convención de Bonn y Acuerdo sobre la Conservación de las Poblaciones de Murciélagos Europeos (EUROBATS) y sus refugios están protegidos por la Convención de Berna y por la Directiva Hábitats. En las últimas décadas se ha producido un claro declive a nivel mundial de las poblaciones de quirópteros; esta situación también ocurre en Portugal, donde 9 especies están catalogadas a nivel nacional de amenazadas (En peligro crítico - CR, En peligro - EN o Vulnerable - VU). La información presentada en este artículo es el resultado de los trabajos de campo realizados entre 2009 y 2011 en el norte y centro de Portugal y aporta un total de 368 citas, resultado de 53 sesiones de trampeo. Los datos corresponden a 22 especies de quirópteros y amplían la distribución conocida de la mayoría de ellas en el área de estudio, al tiempo que aportan algunos datos ecológicos y biométricos de las especies catalogadas. Del total de 368 citas, 90 son nuevas citas de especies para cuadrículas UTM 10x10km

Palabras clave: Quirópteros, lugares de importancia comunitaria, distribución, Península Ibérica, Portugal.

\section{INTRODUCCIÓN}

Los quirópteros son un grupo sumamente diverso que comprende cerca del $20 \%$ de todas las especies europeas de mamíferos (Mitchell-Jones et al. 1999). En el Portugal continental existen un total de 74 especies de mamíferos (Cabral et al. 2005), de las cuales 25 son quirópteros, lo que representa más del $33 \%$. Es también el grupo de mamíferos con mayor grado de amenaza de la fauna portuguesa, dado que 10 especies aparecen recogidas en el Anexo II de la Directiva "Habitats" (92/42/CEE) que relaciona las especies animales y vegetales de interés comunitario para cuya conservación es necesario designar zonas especiales de conservación; y todas las especies de quirópteros del Portugal continental están incluidas en el Anexo IV de la misma Directiva, que relaciona las especies de interés comunitario que requieren una protección estricta. Del listado de especies de quirópteros de Portugal, 9 de ellas (Rhinolophus ferrumequinum, $R$. hipposideros, R. euryale, R. mehelyi, Myotis bechsteinii, $M$. myotis, M. blythii, M. nattereri y Miniopterus schreibersii) están catalogadas a nivel nacional como amenazadas (En peligro crítico - CR, En peligro - EN o Vulnerable - VU) (Cabral et al. 2005), representando el 60\% de las especies de mamíferos amenazados de Portugal. Además, están catalogadas 9 especies más (M. emarginatus, M. mystacinus, Hypsugo savii, Nyctalus leisleri, N. noctula, N. lasiopterus, Barbastella barbastellus, Plecotus auritus y Tadarida 
teniotis) en la categoría Información Insuficiente (DD) (Cabral et al. 2005). Esta categoría es considerada cuando no hay información adecuada para hacer una evaluación directa o indirecta del riesgo de extinción de un taxón, atendiendo a su distribución y/o al estado de su población. Además, están las especies de Eptesicus isabellinus y Myotis escalerai, que no fueron evaluadas en la última edición del Libro Rojo de Vertebrados de Portugal (Cabral et al. 2005), ya que la confirmación de estas especies para Portugal (ICNB, 2010) fue posterior a esta publicación.

El conocimiento de la distribución de los quirópteros en Portugal es aún muy incompleto; reflejo de esto es la ausencia de un atlas nacional de quirópteros (en el cual se está trabajando actualmente). El único documento que presenta una distribución de quirópteros, a pesar de estar representado en cuadriculas UTM de $50 X 50 \mathrm{~km}$, es la "Guía de Mamíferos de Portugal" (Mathias et al., 1999). De hecho, los estudios sobre quirópteros de Portugal se realizaron por primera vez a finales del siglo XIX y Bocage publicó en 1863 la primera lista de especies de quirópteros para Portugal. Otros autores (e.g. Oliveira 1895, Oliveira \& Vieira 1896, Seabra 1900, Nobre 1904, Seabra 1922) fueron añadiendo conocimientos sobre la distribución y composición de las especies presentes en Portugal. Finalmente Themido (1928), relaciona un total de 20 especies para Portugal. A finales de los años 70 se realizaron estudios de recopilación (e. g. Palmeirim et al. 1979), y a principios de los 90 aparece la primera publicación sobre la identificación, morfología y taxonomía de los quirópteros de Portugal (Palmeirim 1990). Posteriormente se han realizado otros estudios de distribución, principalmente dirigidos a las áreas protegidas de Portugal (Bicho 1994, Rainho et al. 1998, Lourenço 2000) como, por ejemplo, el inventario de quirópteros en el Parque Nacional de Peneda-Gerês, Parque Natural Serra da Estrela, Serra de Aires y Candeeiros (Bicho 1995), Parque Natural Arrábida y Serra de S. Mamede (Rainho 1995), Parque Natural Sudoeste Alentejano y Costa Vicentina y Reserva Natural del Estuário del Sado (Franco 1996), Parque Natural da Ria Formosa y Reserva Natural do Sapal de Castro Marim (Rainho 1997), Parque Natural de Sintra-Cascais y Paisage Protegida Arrábida Fosil da Costa da Caparica (Pereira 2000), Parque Natural do Douro Internacinal y Parque Natural do Vale do Guadiana (Rebelo 2001) o en Lugares de Importancia Comunitaria (Braz et al 2009). Este trabajo tiene como principal objetivo aumentar el conocimiento de la distribución de los quirópteros en el Norte y Centro de Portugal y aportar algunos datos ecológicos de las especies catalogadas.

\section{Materiales y MÉTOdos}

La información presentada en este artículo es el resultado de los trabajos de campo realizados entre los meses de abril y octubre de 2009, 2010 y 2011, con un esfuerzo de prospección de 53 sesiones de captura (positivas), en los hábitats más representativos de las zonas montañosas del norte y centro de Portugal (Figura1). Se capturaron un total de 368 ejemplares, todos ellos con redes de niebla situadas tanto en las entradas o inmediaciones de refugios potenciales, como sobre ríos, balsas de agua, caminos y sendas, sotos fluviales, y otras zonas potenciales de paso durante el campeo nocturno (Finnmore \& Richardson 1987, Kunz \& Kurta 1990, Lisón et al. 2010).
La identificación de los ejemplares capturados se realizó en mano mediante la utilización de caracteres morfológicos y de dentición (Dietz \& Helversen 2004, Dietz et al. 2009); además, se tomó información sobre el sexo, edad y estado reproductivo de las hembras, así como las mediciones estándar (peso y longitud del antebrazo). A las especies de los complejos crípticos Eptesicus serotinus/isabellinus, Plecotus auritus auritus/begognae, Myotis daubentoniil/ daunbentonii2, Myotis mystacinus/alcathoe, Myotis cf nattereri/escalerai y Hypsugo savii-1/savii-2, por tener un interés particular para la diferenciación genética (Ibáñez et al. 2006), se les tomó muestras de tejido, con el objetivo de realizar los estudios de ADN mitocondrial de estos complejos. La muestra consiste en biopsias del tejido membranoso de las alas conseguidas con un punch de 2 o $3 \mathrm{~mm}$ de diámetro (en función de la especie); las muestras de tejido fueron preservadas en etanol 70\% (Maniatis et al. 1989) y analizadas (Javier Juste, Estación Biológica de Donana) en el Departamento de Ecología Evolutiva de la Estación Biológica de Doñana.

La identificación de los Pipistrellus pipistrellus y Pipistrellus pygmaeus, fue realizada a partir de características morfológicas descritas en Dietz \& Helversen (2004) y Dietz et al. (2009), además, algunos ejemplares fueron analizados genéticamente, así como todos los pares crípticos mencionados anteriormente capturados en 2009 y 2010.

El área de distribución de las especies se representa cartográficamente en cuadriculas UTM de 10x10 km (Anexo I); geográficamente se representan los lugares de captura en coordenadas UTM kilométricas (Anexo II).

Todas las capturas se han efectuado con las correspondientes autorizaciones (63/2011/CAPT, 1/2010/CAPT y 34/2009/ CAPT) del Instituto de Conservação da Natureza e Biodiversidade (ICNB), autoridad responsable de la emisión de los permisos de capturas.

\section{RESUltados}

Con las 53 sesiones de captura (Tabla 1) se pudieron capturar e identificar 22 especies de murciélagos de las que se aportan 368 citas. La utilización de técnicas moleculares en estudios filogenéticos ha revelado una extraordinaria diversidad de especies crípticas en el ámbito de la Península Ibérica (Ibáñez et al. 2006), con la descripción de taxones endémicos de la Península, como es el caso del M. escalerai, o de especies con distribución ibero-africana, como el caso del E. isabellinus, ambos identificados en el presente estudio.

\section{Familia Rhinolophidae (Gray, 1825)}

Rhinolophus ferrumequinum (Schreber, 1774). Material examinado: Campeã: L48 (28/07/2011), 1 (1ठึ); Vila Cova: L53 (07/10/2011), $1(1 \overbrace{}^{\Uparrow}) . \mathrm{N}=2$.

Rhinolophus hipposideros (Bechstein, 1800). Material examinado: Moimenta: L2 (05/06/2009), 1 (1ふึ); Vila Cova: $\operatorname{L} 53(07 / 10 / 2011), 1\left(1{ }^{\top}\right) . \mathrm{N}=2$.

Rhinolophus euryale Blasius, 1853. Material examinado: Moimenta: L2 (05/06/2009), 1 (1ㅇ). N=2. Nueva cita para la cuadricula UTM $10 \times 10 \mathrm{~km}$ NF73. 


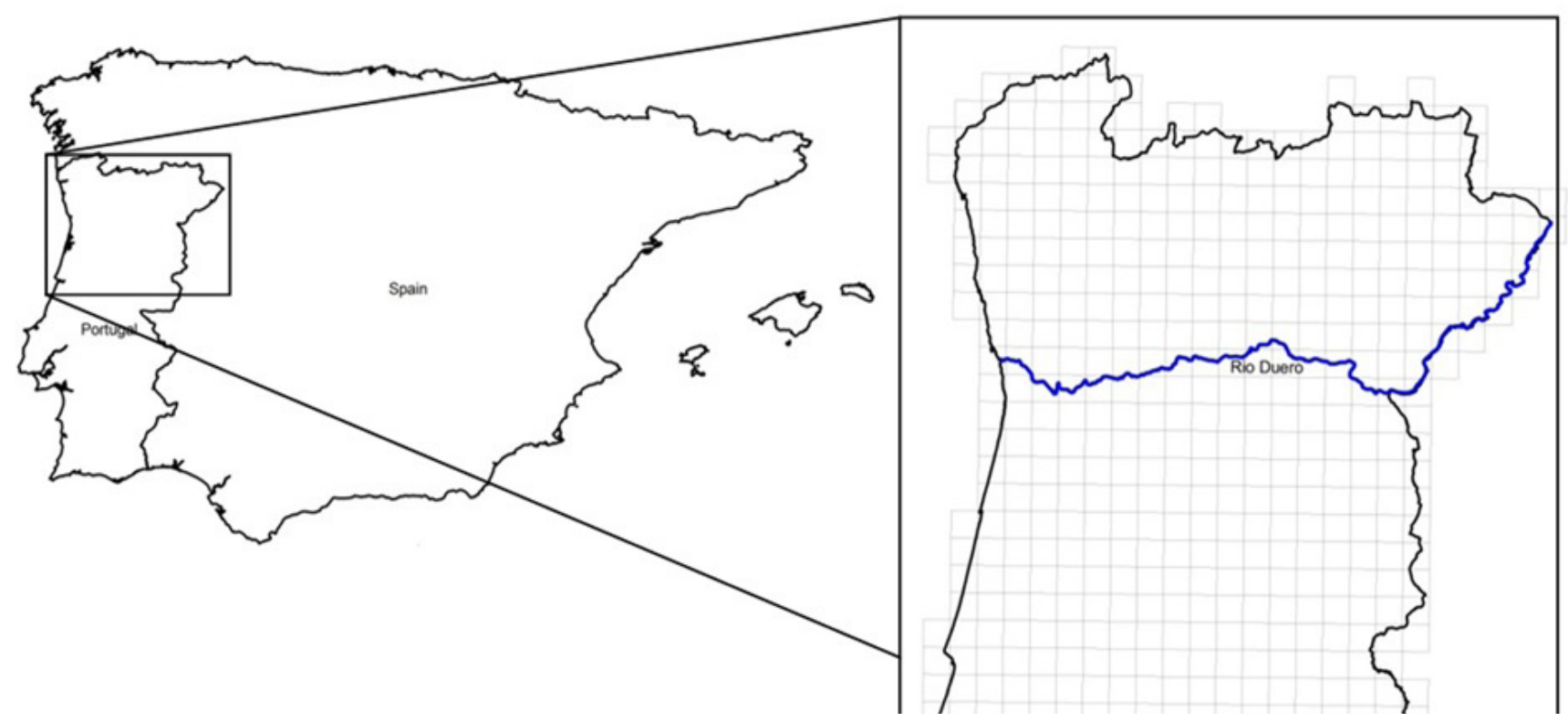

Familia Vespertilionidae (Gray, 1821)

Myotis myotis (Borkhausen, 1797). Material examinado: Lamas de Olo: L1 (30/05/2009), $1\left(1 \delta^{\Uparrow}\right)$; Vila Cova: L21 (23/09/2009), 1 (1ठึ); Lapa dos Dinheiros: L38 $(14 / 08 / 2010), 1\left(1 \jmath^{\Uparrow}\right) . \mathrm{N}=3$. Nuevas citas para las cuadrículas UTM 10X10km PE17 y PF08.

Myotis blythii (Tomes, 1857). Material examinado: Vila Cova: L52 (21/09/2011), 1 (1ठ). $\mathrm{N}=1$.

Myotis emarginatus (Geoffroy, 1806). Material examinado: Vilar de Ferreiros: L13 (29/08/2009), 1 (1q); Campanhó: L18 (13/09/2009), 1 (1 $\left.\delta^{\Uparrow}\right)$; Sazes da Beira: L39 (15/08/2010), $1(1 \precsim)$. $\mathrm{N}=3$. Nueva cita para la cuadrícula UTM 10X10km NF87.

Myotis escalerai (Cabrera, 1904). Material examinado: Campeã: L16 (09/09/2009), 1 (1つ); Penamacor: L27 (03/06/2010), 1 (1ㅇ); Faiões: L34 (03/07/2010), 1 (1ㅇ). Algodres: L35 (09/07/2010), 1 (1ð); Sazes da Beira: L39 (15/08/2010), 2 (1ð̄, 1q); Penamacor: L46 (10/06/2011), 1 (1 9$)$; Campeã: L48 (28/07/2011), 2 (2ð); Vila Cova: L52 (21/09/2011), 3 (3ठึ); Vila Cova: L53 (07/10/2011), 4 (4仓ึ). $\mathrm{N}=16$. Nuevas citas para las cuadrículas UTM 10X10km PG32, NF96, NF97, PF63, PE06 y PE66.

Myotis bechsteinii (Kuhl, 1817). Material examinado: Penamacor: L27 (03/06/2010), $1\left(1 \delta^{\top}\right)$. N=1. Nueva cita para la cuadricula UTM 10X10km PE66.

Myotis daubentonii (Kuhl, 1817). Material examinado: Lamas de Olo: L1 (30/05/2009), 1 (1ठ); Lamas de Olo:

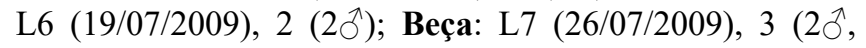

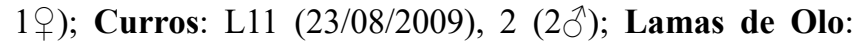
L12 (25/08/2009), 4 (3̧̂, 1ㅇ); Carlão: L19 (15/09/2009), 8 (4今ે, 4q); Chã: L20 (17/09/2009), 1 (19); Vila Cova: L21 (23/09/2009), 1 (1ठ); Ermelo: L22 (24/09/2009), 4 (4仓ึ); Parâmio: L23 (03/10/2009), 1 (1+); Quadrazais: L26 (02/06/2010), 12 (1ठ, 11ㅇ); Penamacor: L28 (04/06/2010), 1 (1ठ); Meimão: L29 (05/06/2010), 1 (1ठึ); Vila Verde da Raia: L36 (06/08/2010), 15 (6へ, 9); Atei: L40 (25/08/2010), 18 (5ô, 13 + $)$; Torgueda: L41
(28/08/2010), 2 (2ð); França: L42 (31/08/2010), 6 (4ठึ, $2 \bigcirc)$; Quadrazais: L47 (11/06/2011), $7(2 \hat{\jmath}, 5 \circ)$; França: L49 (29/07/2011), 3 (1ふ, 2ㅇ); Bragança: L7 (27/08/2011), 5 (3今, 2ᄋ); Vila Cova: L52 (21/09/2011), 1 (1+); Vila Cova: L53 (07/10/2011), $1\left(1 \jmath^{\top}\right) . ~ N=99$. Nuevas citas para las cuadriculas UTM 10X10km PG83, PG32, PG01, NF99, PF29, PF08, PF27, PF37, PE66 y PE65.

Myotis mystacinus (Kuhl, 1817). Material examinado: Lamas de Olo: L06 (19/07/2009), 3 (3ㅇ); Beça: L7 (26/07/2009),

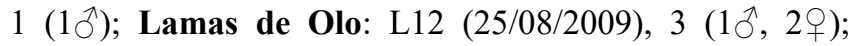
Quadrazais: L47 (11/06/2011), 1 (1ㅇ). N=8. Nuevas citas para las cuadrículas UTM 10X10km PG01, NF98 y PE66.

Pipistrellus pipistrellus (Schreber, 1774). Material examinado: Alijó: L4 (04/07/2009), 2 (10̂, 1 ㅇ); Beça: L7 (26/07/2009), 2 (2ㅇ); Vila Marim; L8 (08/08/2009), 2 (2へ̂); Curros: L11 (23/08/2009), 5 (2へ, 3 ㅇ); Vilar de Ferreiros; L13 (29/08/2009), 2 (2へ); Bilho;L14 (02/09/2009), 2 (1へ,

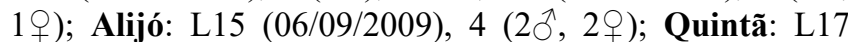
(11/09/2009), 3 (1仓ิ, 2ㅇ); Campanhó: L18 (13/09/2009), 1 (1ठ); Ermelo: L22 (24/09/2009), 3 (1ठ, 2 + $)$; Parâmio: L23 (03/10/2009), 2 (2ð); Quadrazais: L26 (02/06/2010), 3 (3 + ); Penamacor: L 27 (03/06/2010), $1(1 \precsim)$; Penamacor: L28 (04/06/2010), 1 (1ठ); Sanjurge: L33 (02/07/2010), 1

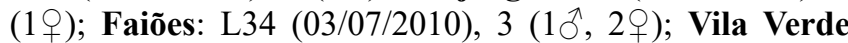
de Raia: L36 (06/08/2010), 1 (1 + ); Padrela de Tazém: L37 (07/08/2010), 1 (1ठึ); Lapa dos Dinheiros: L38 (14/08/2010), $1(1 \overbrace{}^{\Uparrow})$; Sazes da Beira: L39 (15/08/2010), 2 (2) ; Atei: L40 (25/08/2010), 2 (1ठ, 1ㅇ); Torgueda: L41 (28/08/2010), 2 (2+); Vila Verde: L43 (03/09/2010), 1 (1ふ); Quadrazais: L47 (11/06/2011), 5 (1ふึ, 4ㅇ); Zoio: L14 (30/07/2011), 1 (1q); Bragança: L51 (27/08/2011), $7(2 \hat{\bigcirc}, 5 \circ) . \mathrm{N}=62$. Nuevas citas para las cuadrículas UTM 10X10km PG74, PG83, PG22, PG32, PG72, PG01, PG20, NF99, PF29, NF98, NF87, NF97, PF07, PF17, PF27, NF96, PE17, PE06, PE66, PE76 y PE65.

Pipistrellus pygmaeus (Leach, 1825). Material examinado:

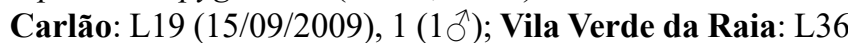
(06/08/2010), 1 (1ð); Sazes da Beira: L39 (15/08/2010), 1 (1)ㅜㅇ Torgueda: L41 (28/08/2010), 1 (1ㅇ); Quadrazais: L47 (11/06/2011), 5 (3ð̄, 2 q); Bragança: L51 (27/08/2011), 
1 (1ठ). $\mathrm{N}=10$. Nuevas citas para las cuadrículas UTM 10X10km PG83, PG32, NF97, PF37, PE06 y PE66.

Pipistrellus kuhlii (Kuhl, 1817). Material examinado: Ermelo: L5 (11/07/2009), 1 (1 +); Curros: L11 (23/08/2009), 1 (1)); Vilar de Ferreiros: L13 (29/08/2009), 1 (1q); Campanhó: L18 (13/09/2009), 1 (19); Penamacor: L28 (04/06/2010), 3 (3ㅇ); Algordes: L31 (20/06/2010), 1 (1q); Algordes: L35 (09/07/2009), 2 (10, 1 ㅇ); Vilar de Maçada: L44 (10/05/2011), 1 (1 + ). $\mathrm{N}=11$. Nuevas citas para las cuadrículas UTM 10X10km PF29, NF87, PF27, PF63 y PE65.

Hypsugo savii (Bonaparte, 1837). Material examinado: Ermelo: L5 (11/07/2009), 3 (2へ, 1 q); Lamas de Olo: L6 (19/07/2009), 1 (19); Vila Marim: L8 (08/08/2009), 2 (2ㅇ); Curros: L11 (23/08/2009), 2 (1ठ, 1우); Campanhó: L18 (13/09/2009), 2 (1ふ̄, 1ㅇ); Parâmio: L23 (11/07/2009), 1 (1 + ); Chã: L25 (22/05/2010), 1 (1ठ); Penamacor: L27 (03/06/2010), 1 (1ठ); Sanjurge: L33 (02/07/2010), 2 (2ㅇ); Faiões: L34 (03/07/2010), 1 (1 + ); Lapa dos Dinheiros: L38 (14/08/2010), $1(1 \circlearrowleft)$; Sazes da Beira: L39 (15/08/2010), 1 (1ð); Penamacor: L46 (10/06/2011), $1(1 \precsim)$. N=19. Nuevas citas para las cuadrículas UTM 10X10km PG22, PG32, PF29, NF87, PF07, PF27, PE17 y PE66.

Nyctalus leisleri (Kuhl, 1817). Material examinado: Beça: L7 (26/07/2009), 1 (1ठึ); Curros: L11 (23/08/2009), 1 (19); Bilhó: L14 (02/09/2009), 2 (2ᄋ); Quintã: L17 (11/09/2009), 2 (1ڤ̂, 1ㅇ); Parâmio: L23 (03/10/2009), 3 (3ठ); Chã: L24 (05/04/2010), 1 (1 + ); Sanjurge: L33 (02/07/2010), 2 (2)); Lapas dos Dinheiros: L38 (14/08/2010), 2 (2ð); Sazes da Beira: L39 (15/08/2010), 1 (1ð); Torgueda: L41 (28/08/2010), 1 (1ð); Zoio: L50 (30/07/2011), 1 (1ㅇ). N=17. Nuevas citas para las cuadrículas UTM 10X10km PG22, PG72, PG01, PF29, PF27, PE17 y PE06.

Nyctalus noctula (Schreber, 1774). Material examinado: Sanjurge: L33 (02/07/2010), 1 (1우). N=1. Nueva cita para la cuadricula UTM $10 X 10 \mathrm{~km}$ PG22.

Eptesicus serotinus (Schreber, 1774). Material examinado: Ermelo: L5 (11/07/2009), 7 (4仓, 3 ㅇ); Lamas de Olo: L6 (19/07/2009), 1 (1ठึ); Vilar de Ferreiros: L13 (29/08/2009), 3 (3 + ); Campanhã: L16 (09/09/2009), 1 (1ð); Quintã: L17 (11/09/2009), 2 (1ठ, 1ㅇ); Vila Cova: L21 (23/09/2009),

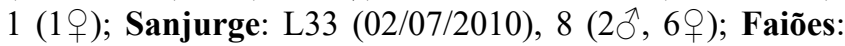
L34 (03/07/2010), 1 (1); Lapa dos Dinheiros: L38 (14/08/2010), 3 (1仓ิ, 2 ㅇ); Sazes da Beira: L39 (15/08/2010), 1 (1ㅇ); Torgueda: L39 (15/08/2010), 1 (1 $\overbrace{}^{\lambda})$; Vilar de Ferreiros: L45 (26/05/2011), $1\left(1 \delta^{\Uparrow}\right)$. $N=30$. Nuevas citas para las cuadrículas UTM 10X10km PG22, PG32 y NF96.

Eptesicus isabellinus (Temminck, 1840). Material examinado: Ermelo: L35 (09/07/2010), 1 (1ठ̂). N=1. Nueva cita para la cuadricula UTM 10X10km PF63.

Barbastella barbastellus (Schreber, 1774). Material examinado: Bilhó: L10 (20/08/2009), 1 (1ð); Vila cova: L21 (23/09/2009), 1 (1ठ); Penamacor: L27 (03/06/2010),

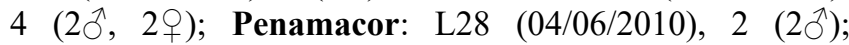
Padrela de Tazém: L37 (07/08/2010), 3 (1へ̂, 2ㅇ); Zoio: L50 (30/07/2011), 1 (1ㅇ); Vila Cova: L52 (21/09/2011),
9 (6ð, 3 ㅇ). $\mathrm{N}=21$. Nuevas citas para las cuadrículas UTM 10X10km PG72, PG20, NF88, PE66 y PE65.

Plecotus austriacus (Fischer, 1829). Material examinado: Alijó: L3 (21/06/2009), 4 (1今̄, 3年); Ermelo: L5 (11/07/2009), 1 (1q); Vila Marim: L9 (14/08/2009), 7 (3仓ै, 4ㅇ); Vilar de Ferreiros: L13 (29/08/2009), 2 (2ㅇ); Alijó: L15 (06/09/2009), 12 (5ठ, 7ㅇ); Penamacor: L27 (03/06/2010), 7 (3ðे, 4ㅇ); Meimão: L29 (05/06/2010), 1 (1ㅇ); Algodres: L30 (19/06/2010), 1 (1); Algodres: L31 (20/06/2010), 1 (1ठึ); Padrela de Tazém: L37 (07/08/2010), $1(1 \circlearrowleft)$; Lapa dos Dinheiros: L38 (14/08/2010), 2 (2ð); Vilar Maçada: L44 (10/05/2010), 4 (2仓, 2 ㅇ); Penamacor: L46 (10/06/2011), 2 (1ð,, 1); Zoio: L50 (30/07/2011), 1 (1ठ); Vila Cova: L53 (07/10/2011), 2 (2ð). N=48. Nuevas citas para las cuadriculas UTM 10X10km PG72, PG20, NF97, PF27, PF63, PE66 y PE65.

Plecotus auritus (Linnaeus, 1758). Material examinado: Penamcor: L27 (03/06/2010), 4 (1 $\widehat{\jmath}, 3$ + $)$; Meimão: L29 (05/06/2010), 1 (1ㅇ); Padrela de Tazém: L37 (07/08/2010), $1(1 \circlearrowleft)$; Quadrazais: L47 (11/06/2011), 1 (1q); Zoio: L50 (30/07/2011), 1 (1థ); Vila Cova: L53 (07/10/2011), 1 (1ठ). $\mathrm{N}=9$. Nuevas citas para las cuadrículas UTM 10X10km PG20 y PE65.

\section{Familia Miniopteridae (Bonaparte, 1837)}

Miniopterus schreibersii (Kuhl, 1817). Material examinado: Vila Marim: L8 (08/08/2009), 1 (1); Vila Cova: L21 (23/09/2009), 4 (4ठึ); Vila Cova: L52 (21/09/2011), 1 (1 $\left.\delta^{\Uparrow}\right)$; Vila Cova: L53 (07/10/2011), 1 (1ㅇ). $\mathrm{N}=7$. Nueva cita para la cuadricula UTM $10 X 10 \mathrm{~km}$ PF07.

\section{Discusión}

Las especies capturadas con mayor frecuencia fueron $M$. daubentonii $(\mathrm{n}=99)$ y P. pipistrellus $(\mathrm{n}=62)$. De hecho, estas especies están entre las más comunes y de mayor distribución en Europa (Mitchell-Jones et al. 1999). M. daubentonii utiliza los bosques de ribera, que abundan principalmente en el Norte de Portugal, como hábitat preferencial (Warren et al. 2000); por el contrario, P. pipistrellus es una especie más generalista (Davidson-Wattsa et al. 2006) que caza en todo tipo de hábitats, incluso los más humanizados.

Las cuadrículas con mayor número de especies han sido las UTM 10X10 NF97, PE66 y la NF98, con 14, 10 y 9 especies respectivamente (Figura 2); no obstante, el mayor número de especies capturadas en estas cuadrículas no se debe a que posean una mayor o menor diversidad en comparación con otras, sino al mayor esfuerzo de muestreo realizado en ellas. De hecho la media de captura fue de 7,01 individuos y 3,05 especies por sesión de trampeo.

El número de especies de quirópteros presentes en Europa ha aumentado un $20 \%$ debido a la semejanza morfológica entre algunas especies (Evin et al. 2009). Uno de los casos más recientes es el de $M$. escalerai, taxón más abundante y frecuente (del complejo M. nattereri) en la Península Ibérica y de hábitos cavernícolas (Ibáñez et al. 2006). De hecho, todas las capturas hechas de este complejo, resultaron ser de Myotis escalerai, capturados entre los 524 y 1227 m.s.n.m en entradas de minas o en su proximidad. 
Algunas especies consideradas como raras o muy localizadas en Portugal con la escasa información de la que se dispone para ellas, como M. emarginatus o B. barbastellus (Cabral et al. 2005), fueron capturadas en localidades diversas, tanto en el norte como en el centro de Portugal; y en hábitats atípicos, como es el caso de B. barbastellus, que suele aparecer casi exclusivamente en bosques caducifolios (Galán et al. 2005). Los dados recientes de presencia de Nyctalus noctula en Portugal son muy escasos y apenas para el Norte e Centro de Portugal, resumiendo-se a una observación de un ejemplar hibernando en un túnel ferroviario (LEA, 2009) y un par de ejemplares muertos en parques eólicos (ICNB, 2010), en el caso del ejemplar e Nyctalus noctula descrito en este articulo, una hembra sin indicios de estar preñada o lactante, identificado a partir de sus características morfológicas (dimensión del antebrazo), fue capturado en una rede de niebla de $12 \mathrm{~m}$ que se coloco atravesando un punto de agua (tanque de 15x9 metros) creado para abastecer helicópteros de combate a incendios, inserido en bosque de Quercus suber disperso. De hecho la escasez de citas podría deberse más a un déficit de prospección o métodos de muestreo que a una escasez real, por lo que una diversificación de técnicas (detección de ultrasonidos, prospección de refugios y captura con redes de niebla) incrementaría notablemente su distribución conocida.

Otro dato muy interesante se refiere a Eptesicus isabellinus, de distribución ibero-africana, que ha sido recientemente separada de su especie gemela (Eptesicus serotinus) gracias a los resultados de estudios genéticos (Juste et al. 2009), ya que es la observación más al norte de la Península Ibérica; dato a tener en cuenta para la definición de su distribución ibérica. A pesar de ser una especie abundante, que convive con el hombre en zonas urbanas, debido a su interés como endemismo Ibérico debería tener algún grado de protección (Lisón et al. 2011). El conocimiento de la distribución de los quirópteros en Portugal es aún muy incompleto, dado que apenas algunas áreas protegidas de Portugal y Lugares de Importancia Comunitaria, poseen información sobre este grupo faunístico. Los muestreos de captura no fueron hechos sistemáticamente; no obstante, los resultados permiten mejorar el conocimiento y llenar algún vacío de información de este grupo faunístico aportando 90 nuevas citas de especies para cuadriculas UTM 10X10km, contribuyendo a dibujar los patrones generales de distribución en Portugal, en especial para las especies con Información Insuficiente (DD) (Cabral et al. 2005) y su revisión.

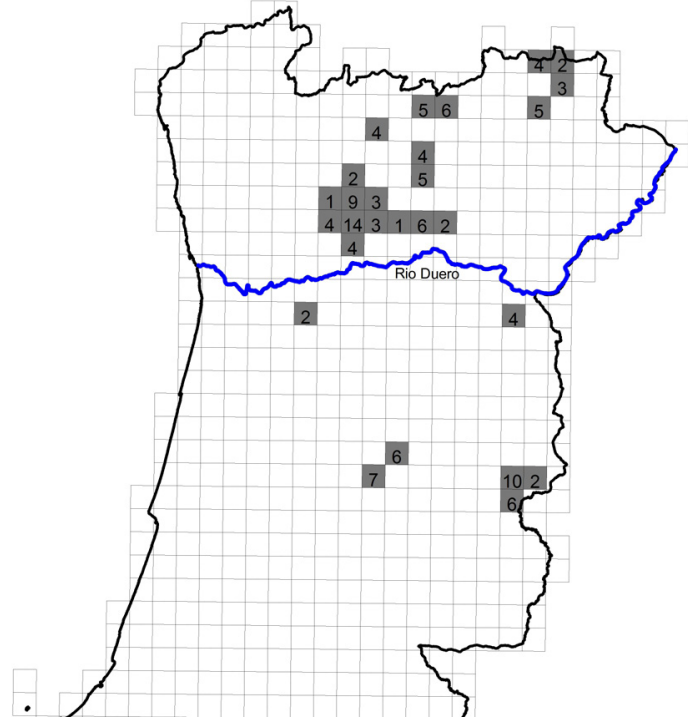

Fig. 1. Riqueza específica de las cuadrículas muestreadas 


\begin{tabular}{|c|c|c|c|c|c|c|c|c|c|c|c|c|c|c|c|c|c|c|c|c|c|}
\hline Espécies & 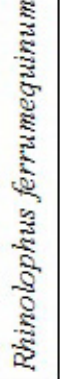 & 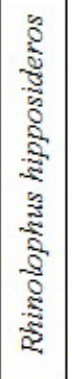 & 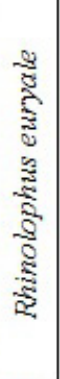 & 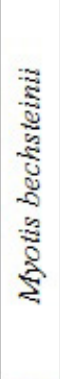 & 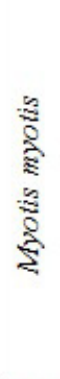 & 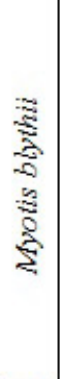 & 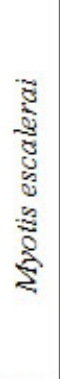 & 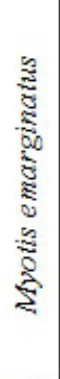 & 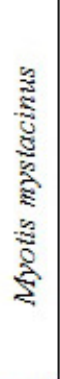 & 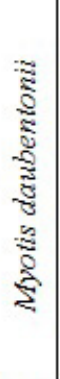 & 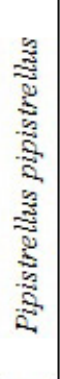 & 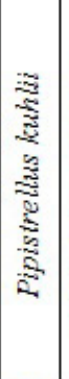 & 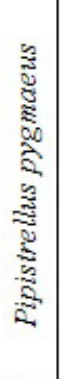 & 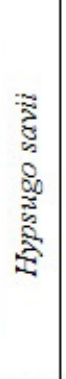 & 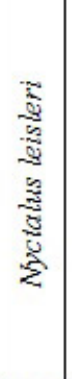 & 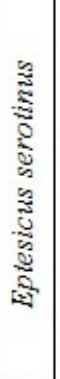 & 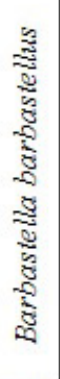 & 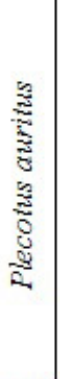 & 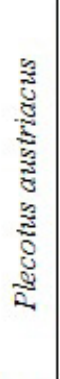 & 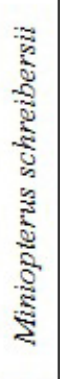 & Total \\
\hline LVVP & $\mathrm{VU}$ & $\mathrm{VU}$ & $\mathrm{CR}$ & EN & $\mathrm{VU}$ & $\mathrm{CR}$ & VU & $\mathrm{DD}$ & $\mathrm{DD}$ & $\mathrm{LC}$ & $\mathrm{LC}$ & LC & $\mathrm{LC}$ & $\mathrm{DD}$ & DD & LC & $\mathrm{DD}$ & $\mathrm{DD}$ & $\mathrm{LC}$ & VU & \\
\hline DH & $\begin{array}{l}\geq \\
\dot{m} \\
\bar{m} \\
\dot{m}\end{array}$ & $\begin{array}{l}\geq \\
\dot{m} \\
\bar{\emptyset}\end{array}$ & $\begin{array}{l}\geq \\
\dot{m} \\
\bar{\emptyset} \\
\dot{m}\end{array}$ & $\begin{array}{l}\geq \\
\dot{\infty} \\
\bar{\emptyset}\end{array}$ & $\begin{array}{l}\geq \\
\dot{m} \\
\bar{\prime} \\
\dot{m}\end{array}$ & $\begin{array}{l}\geq \\
\dot{\infty} \\
\bar{\emptyset} \\
\dot{m}\end{array}$ & $\underset{\dot{m}}{\geq}$ & $\begin{array}{l}\geq \\
\dot{m} \\
\bar{\emptyset}\end{array}$ & $\underset{\dot{m}}{\geq}$ & $\underset{\dot{m}}{\gtrless}$ & 方 & $\underset{\dot{m}}{\geq}$ & 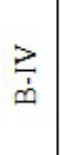 & $\underset{\dot{m}}{Z}$ & $\underset{\dot{n}}{\geq}$ & $\underset{\infty}{m}$ & $\begin{array}{l}\vec{\prime} \\
\dot{m} \\
\bar{\prime} \\
\dot{m}\end{array}$ & $\underset{\dot{m}}{Z}$ & 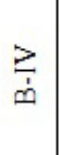 & $\begin{array}{l}\frac{z}{\dot{\prime}} \\
\dot{m} \\
\dot{\bar{\omega}}\end{array}$ & \\
\hline PNA & & & & & $\mathrm{X}$ & & & & $\mathrm{X}$ & $\mathrm{X}$ & $\mathrm{X}$ & $\mathrm{X}$ & & $\mathrm{X}$ & $\mathrm{X}$ & $\mathrm{X}$ & $\mathrm{X}$ & & $\mathrm{X}$ & $\mathrm{X}$ & 11 \\
\hline PNM & & & & & & & & & & $\mathrm{X}$ & $\mathrm{X}$ & & & $\mathrm{X}$ & $\mathrm{X}$ & & & & & & 4 \\
\hline PNSE & & & & & $\mathrm{X}$ & & $\mathrm{X}$ & $\mathrm{X}$ & & & $\mathrm{X}$ & & $\mathrm{X}$ & $\mathrm{X}$ & $\mathrm{X}$ & $\mathrm{X}$ & & & $\mathrm{X}$ & & 9 \\
\hline RNSM & & & & $\mathrm{X}$ & & & $\mathrm{X}$ & & $\mathrm{X}$ & $\mathrm{X}$ & $\mathrm{X}$ & $\mathrm{X}$ & $\mathrm{X}$ & $\mathrm{X}$ & & & $\mathrm{X}$ & $\mathrm{X}$ & $\mathrm{X}$ & & 11 \\
\hline PTCON0002 & & & & & & & & & & $\mathrm{X}$ & $\mathrm{X}$ & & & $\mathrm{X}$ & $\mathrm{X}$ & & $\mathrm{X}$ & $\mathrm{X}$ & $\mathrm{X}$ & & 7 \\
\hline PTCON0003 & $\mathrm{X}$ & $\mathrm{X}$ & & & $\mathrm{X}$ & $\mathrm{X}$ & $\mathrm{X}$ & $\mathrm{X}$ & $\mathrm{X}$ & $\mathrm{X}$ & $\mathrm{X}$ & $\mathrm{X}$ & $\mathrm{X}$ & $\mathrm{X}$ & $\mathrm{X}$ & $\mathrm{X}$ & $\mathrm{X}$ & $\mathrm{X}$ & $\mathrm{X}$ & $\mathrm{X}$ & 18 \\
\hline PTCON0004 & & & & $\mathrm{X}$ & & & $\mathrm{X}$ & & $\mathrm{X}$ & $\mathrm{X}$ & $\mathrm{X}$ & $\mathrm{X}$ & $\mathrm{X}$ & $\mathrm{X}$ & & & $\mathrm{X}$ & $\mathrm{X}$ & $\mathrm{X}$ & & 11 \\
\hline PTCON0014 & & & & & $\mathrm{X}$ & & $\mathrm{X}$ & $\mathrm{X}$ & & & $\mathrm{X}$ & & $\mathrm{X}$ & $\mathrm{X}$ & $\mathrm{X}$ & $\mathrm{X}$ & & & $\mathrm{X}$ & & 9 \\
\hline PTCON0025 & & $\mathrm{X}$ & $\mathrm{X}$ & & & & & & & & & & & & & & & & & & 2 \\
\hline
\end{tabular}

Tabla 1. Listado de especies, relacionadas por Parques y Reservas Naturales y Lugares de Interese Comunitario (LIC) con las categorías de la Directiva Habitas (HD) y las de conservación de acuerdo con el Livro Vermelho de Vertebrados de Portugal (LVVP) (Cabral et al., 2005). Las abreviaturas de los Parques y Reservas Naturales son las siguientes: PNA (Parque Natural do Alvão); PNM (Parque Natural de Montesinho); PNSE (Parque Natural da Serra da Estrela) y RNSM (Reserva Natural da Serra da Malcata). Las abreviaturas de los LICs son las siguientes: PTCON0002 (LIC Montesinho/Nogueira), PTCON0003 (LIC Alvão/Marão), PTCON00004 (LIC Malcata), PTCON0014 (LIC Serra Estrela) y PTCON0025 (LIC Serra de Montemuro).

\section{Agradecimientos}

Deseo expresar mi agradecimiento a todos los que me acompañaron en las sesiones de capturas: Anabela Paula, Antero Lopes, Carlos Rodrigues, Cristina Lopez, David Lopes, Emanuel Ribeiro, Fernanda Pereira, Hélia Gonçalves, Isabel Passo, Jacinta Martins, Joana Medeiros, João Gaiola, João Paula, Luís Braz, Marco Fachada, Paulo Travassos. Un agradecimiento especial a la Estación Biológica de Doñana, en particular a Javier Juste y todo su equipo por la confirmación genética de algunos ejemplares. Muy especialmente quiero mostrar mi agradecimiento a Roberto Hermida, por su valiosa revisión y comentarios al manuscrito original y por me meter o "bichinho" de esto de los murciélagos.

\section{REFERENCIAS}

BICHO, S. 1994. Inventariação de morcegos em Áreas Protegidas: Parque Natural do Alvão e Parque Natural de Montesinho. Relatório interno. ICN.

BichO, S. 1995. Inventariação dos morcegos presentes no Parque Nacional da Peneda-Gerês e nos Parques Naturais da Serra da Estrela e Serras de Aire e Candeeiros. Estudo dos biótopos de alimentação. Relatório interno. ICN.
Braz, L,. H. Gonçalves, P. Barros \& P. Travassos 2009. First record of Bechstein's bat (Myotis bechsteinii Kuhl, 1817) at North of Portugal and new specie for the site of comunitary importance, Alvão-Marão. Galemys 21(1): 71-75.

Cabral M. J, Almeida, J , Almeida, P. R., Dellinger, T., FerranddeAlmeida, N., Oliveira,M.E.,Palmeirim, J. M., QueIroz, A. I., Rogado, L. \& SAntos-Reis, M. 2005. Livro Vermelho dos Vertebrados de Portugal. $2^{\mathrm{a}}$ ed. Instituto da Conservação da Natureza/Assírio \& Alvim. Lisboa, 660 pp.

Davidson-Wattsa, I., Wallsc, S. \& Jonesd, G. 2006. Differential habitat selection by Pipistrellus pipistrellus and Pipistrellus pygmaeus identifies distinct conservation needs for cryptic species of echolocating bats. Biological conservation 33:118-127. DOI: https://dx.doi.org/10.1016/j. biocon.2006.05.027

Dietz, C. \& Helversen, O. V. 2004. Illustrated identification key to the bats of Europe.

Dietz, C., Helversen, O. V. \& NiLl, D. 2009. Bats of Britain, Europe \& Northwest Africa. A \& C Black Publishers Ltd. 
Evin, A., Lecoq, V., Durand, M. O., Tillon, L. \& Pons, J. M. 2009. A new species for the French bat list: Myotis escalerai (Chiroptera : Vespertilionidae). Mammalia 73: 142-144. DOI: https://dx.doi. org/10.1515/MAMM.2009.030

FinNemoRe, M. \& Richardson, P. W. 1999. Catching bats. In: A.J. Mitchell-Jones and A.P. McLeish, Editors, The Bat Workers' Manual, Joint Nature Conservation Committee, Peterborough, UK. pp. 33-38.

Franco, C. M. 1996. Inventariação dos morcegos e determinação dos seus biótopos de alimentação na Reserva Natural do Estuário do Sado e no Parque Natural do Sudoeste Alentejano e Costa Vicentina. Relatório interno. ICN.

Galán, P. 2000. Primeros datos sobre el Murciélago bigotudo Myotis mystacina (Kuhl, 1817) en Galicia. Galemys, 12 (1): 41-47.

Galán, P., Barros, A., Cerqueira, F. \& Seage, R. 2005 Datos sobre distribución de quirópteros en el norte de Galicia .Galemys 17 (1-2)

IbÁÑEZ,C,García-MudarRa,J.L.,Ruedi,M.,Stadelmann,B. \& JUSTE, J. 2006. The Iberian contribution to cryptic diversity in European bats. Acta Chiropterologica 8 (2): 277-297. DOI: http://dx.doi.org/10.3161/17335329(2006)8[277:TICTCD]2.0.CO;2

ICNB 2010. Agreement on the Conservation of Populations of European Bats. Report on implementation of the Agreement in Portugal. 2010 / 6 MoP. Instituto da Conservação da Natureza e Biodiversidade.

ICNB (2010) Avaliação do efeito dos parques eólicos sobre os morcegos em Portugal continental (análise dos dados disponíveis em Outubro de 2009). Instituto da Conservação da Natureza e da Biodiversidade. Relatório não publicado.

Juste, J,BILGIN, R, MuÑoz, J\&IBÁÑEZ, C. 2009. Mitochondrial DNA signatures at different spatial scales: from the effects of the Strait of Gibraltar to population structure in the meridional serotine bat (Eptesicus isabellinus). Heredity 103: 178-187. DOI: https:// dx.doi.org/10.1038/hdy.2009.47

Kunz, T .H. \& Kurta, A. 1990. Capture methods and holding devices. In: KUNZ, T.H., Ecological and Behavioral Methods for the Study of Bats, Washington, D.C. London: Smithsonian Institution Press. pp. 1-29.

LEA (2009). Estudo Complementar da Utilização dos Túneis Ferroviários da Linha do Tua pelos Quirópteros no Âmbito do Aproveitamento Hidroeléctrico da Foz do Tua (AHFT). Laboratório de Ecologia Aplicada (LEA), Universidade de Trás-os-Montes e Alto Douro (UTAD). Vila Real. Relatório Final, 54 pp.
Lisón, F., Aledo, E. \& Calvo, J. F. 2011. Los murciélagos (Mammalia: Chiroptera) de la Región de Murcia (SE España): distribución y estado de conservación. Anales de Biologia 33: 79-92, 2011

Lisón, F., Yelo, N. D., Haz, A. \& Calvo, J. F. 2010. Contribución al conocimiento de la distribución de la fauna quiropterológica de la Región de Murcia. Galemys 22 (1): 11-28.

LOURENÇO, S. I. 2000. Inventariação de morcegos e determinação dos seus biótopos de alimentação em Áreas Protegidas. Relatório interno. ICN.

Maniatis, T., Fritsh, F. E \& SAmbrook, J. 1989. Molecular cloning: a laboratory manual. 2nd edition. Cold Spring Harbor Laboratory Press. New York.

Mathias,M.L.,Ramalhinho,M.G.,Palmeirim,J.,Rodrigues, L., Rainho, A., Ramos, M. J., Santos-Reis, M., Petrucci-Fonseca, F., Oom, M. M., Cabral, M. J., Borges, J. F., Guerreiro, A., Magalhães, C. \& PereirA, M. 1999. Guia dos Mamíferos Terrestres de Portugal Continental, Açores e Madeira. ICN. Lisboa. 199pp.

Mitchell-Jones, A. J., Bogdanowicz, W., KryštufeK, B, Reijnders, P. J. H., Spitzenberger, F., Stubbe, M., Thissen, J. B. M., Vohralík, V., \& Zima, J. 1999. Atlas of European mammals. Academic Press, London. 484 pp.

Nobre, A. F. 1904. Fauna Portugueza-VertebradosMammiferos. Annuario da Academia Polytechnica do Porto, pp. 86-89.

Olveira, M. P. \& Vieira, L. 1896. Catalogo dos mamíferos de Portugal. Annaes. de Sciencias Naturaes Porto. 3:9-16.

OlveIRA, M. P. 1895. Tabela dicotómica para a determinação dos mamíferos de Portugal. Annaes de Sciencias Naturaes Porto 2:200-208.

Palmeirim, J. M., Ramos, M. J. \& Dias, D. 1979. Bats from Portugal in the collection of Museu Bocage. Arq. Museu Bocage, (série 2) 7(4):53-66.

Palmeirim, J.M. 1990. Bats from Portugal: zoogeography and systematics. Miscellaneous. Publications of the Kansas University Museum of Natural History 82, 53pp.

Pereira, M. J. R. 2000. Inventariação das espécies e abrigos de morcegos no PNSC e PPAFCC/LA. Determinação de biótopos de alimentação de algumas espécies de morcegos. Relatório interno. ICN.

RAINHO, A. 1995. Inventariação das espécies e dos abrigos nos Parques Naturais da Arrábida e da Serra de S. Mamede, e determinação dos biótopos de alimentação de algumas espécies de morcegos. Relatório interno. ICN. 
RAINHO, A. 1997. Estudo preliminar dos morcegos presentes no Parque Natural da Ria Formosa, Zona de Protecção especial do Estuário do Tejo e Reserva Natural do Sapal de Castro Marim. Relatório técnico. ICN.

Rainho, A., Rodrigues, L., Bicho, S., Franco, C. \& Palmeirim, J. M. 1998. Morcegos das Áreas Protegidas Portuguesas (I). Estudos de Biologia e Conservação da Natureza, 26. 118 pp.

REBELO, H. 2001. Inventariação dos morcegos e determinação dos biótopos de alimentação no Parque Natural do Douro Internacional e Parque Natural do Vale do Guadiana. Relatório interno. ICN.

Rodrigues, L., Alves, P., Silva, B. \& Pereira, M. J. 2011. Chave ilustrada simplificada de identificação das espécies de morcegos presentes em Portugal Continental - Versão 1.1.Publicação Electrónica. Versão 1.1. publicada em 1 de Setembro de 2011
Seabra, A .F. 1922. Notes sur les Chiropteres du Portugal. Bull. Soc. Portugaise Sci. Nat. 9:49-50.

Seabra, A. F. 1900. Mamíferos de Portugal no Museu de Lisboa. Jornal de Sciencias Mathematicas Physicas e Naturaes. 6:90-115.

Themido, A. A. 1928. Catalogue des chirópteres existants dans les colections du Museum Zoologique de Coimbra. Memórias e Estudos do Museu Zoológico da Universidade de Coimbra, Série I, 16.

Warren, R. D., Waters, D. A., Altringham, J. D., Bullock, D. J. 2000. The distribution of Daubenton's bats (Myotis daubentonii) and pipistrelle bats (Pipistrellus pipistrellus) (Vespertilionidae) in relation to small-scale variation in Ríoine habitat. Biological Conservation, 92: 85-91. 


\section{Anexo I}

Mapas con la distribución de las cuadrículas en que han sido capturadas las diferentes especies. Las cuadrículas a negro representan nuevas citas para las especies, a gris las cuadrículas ya descritas para las especies.

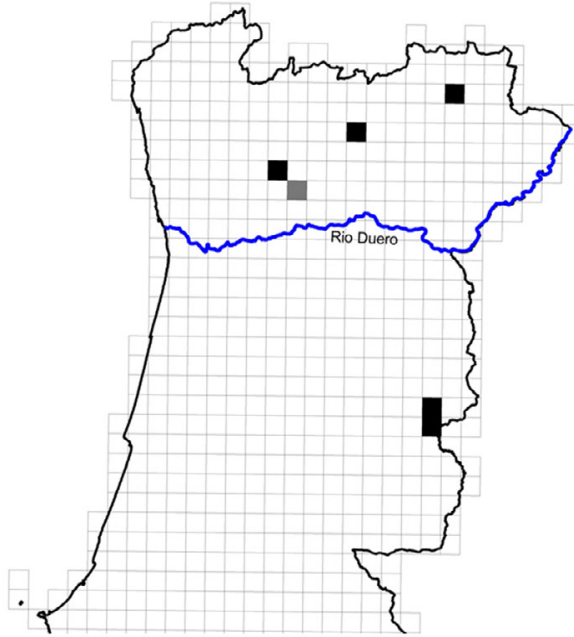

Barbastella barbastellus

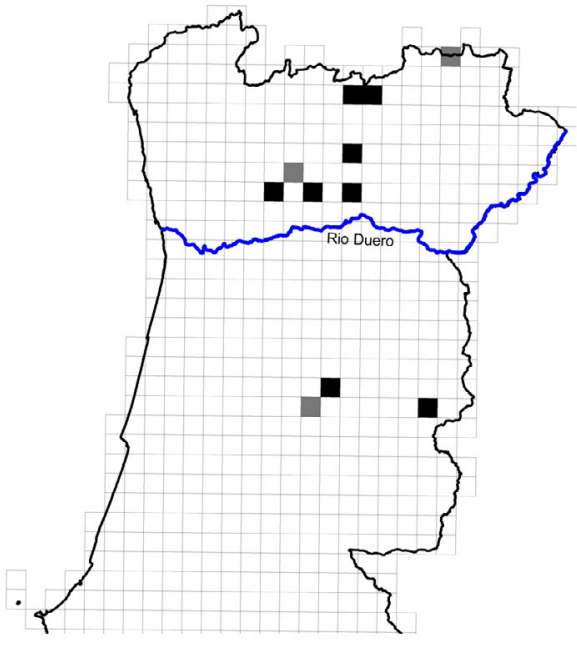

Hypsugo savii

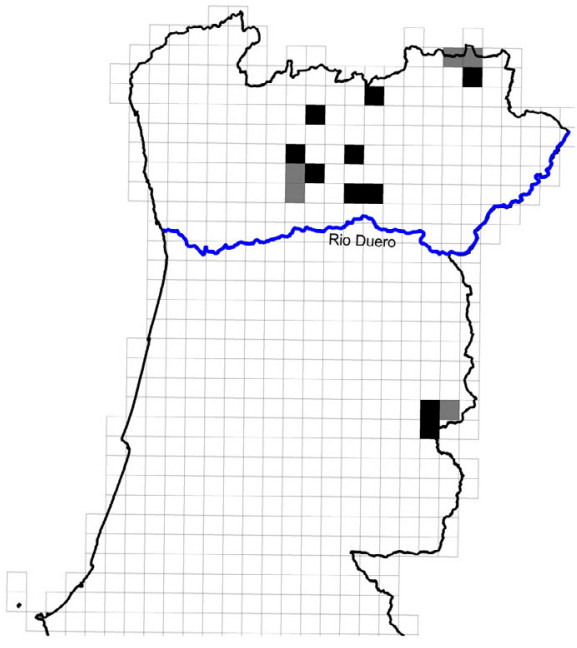

Myotis daubentonii

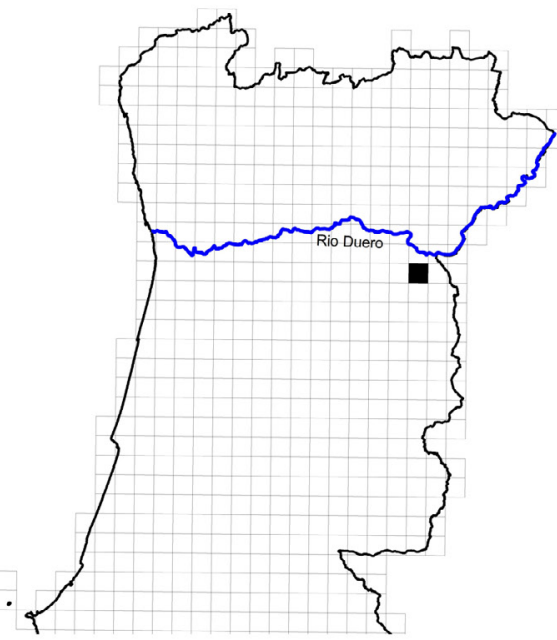

Eptesicus isabellinus

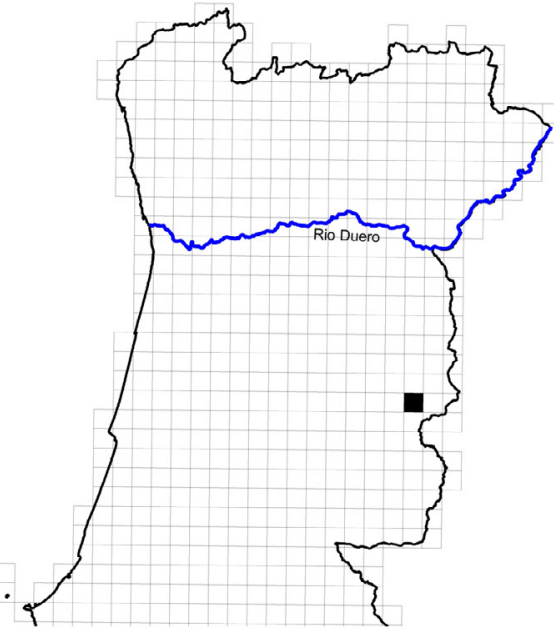

Myotis bechsteinii

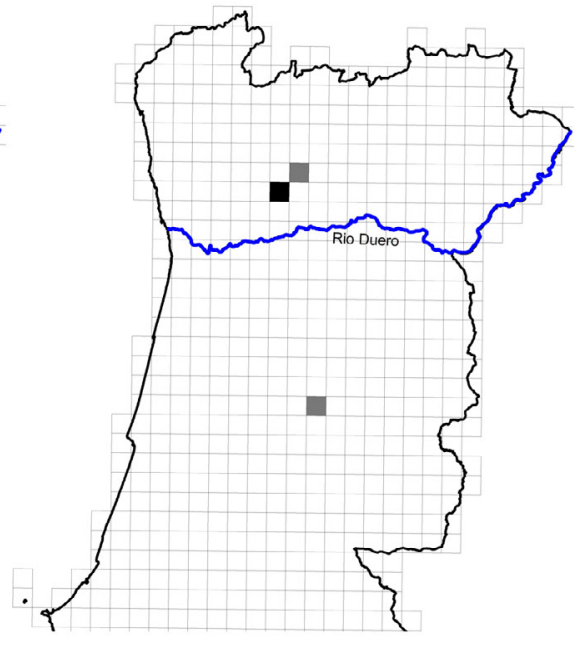

Myotis emarginatus

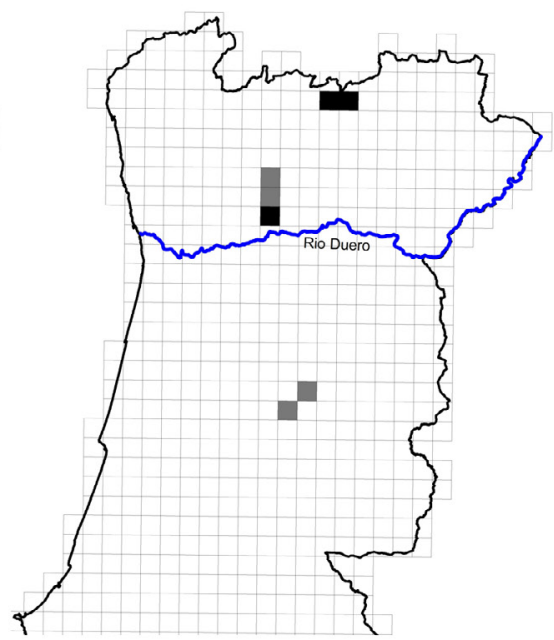

Eptesicus serotinus

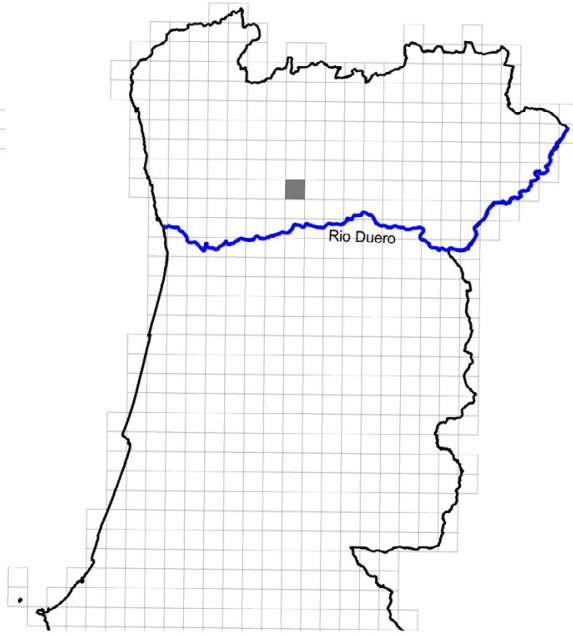

Myotis blythii

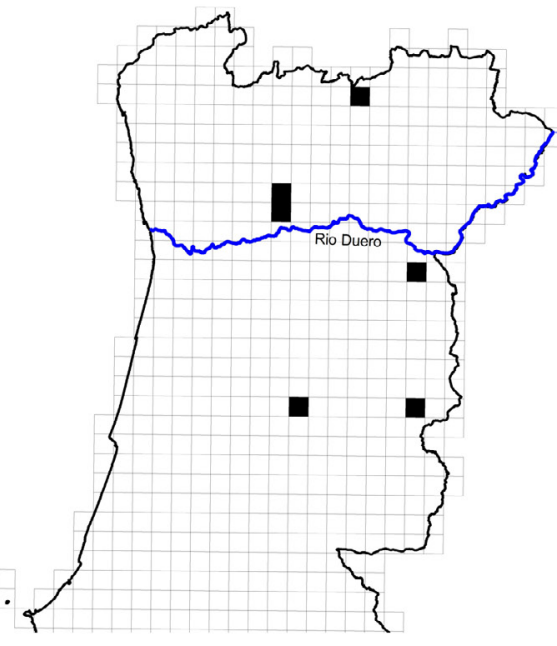

Myotis escalerai 


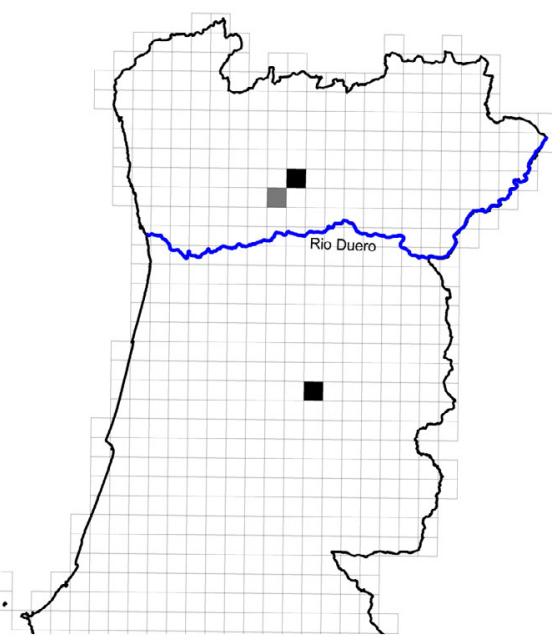

Myotis myotis

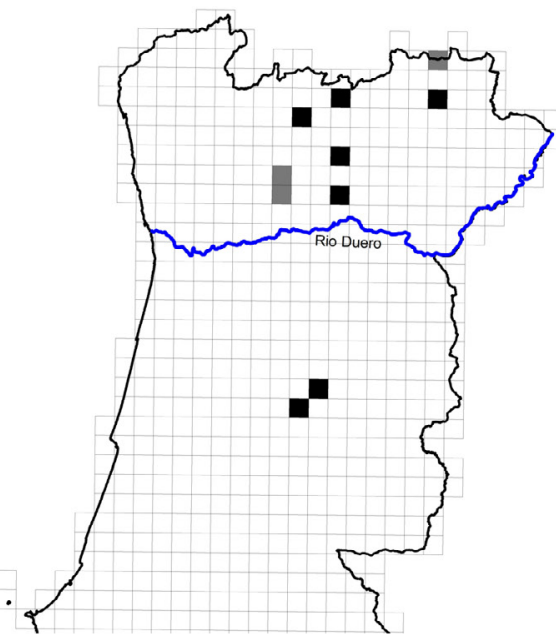

Nyctalus leisleri

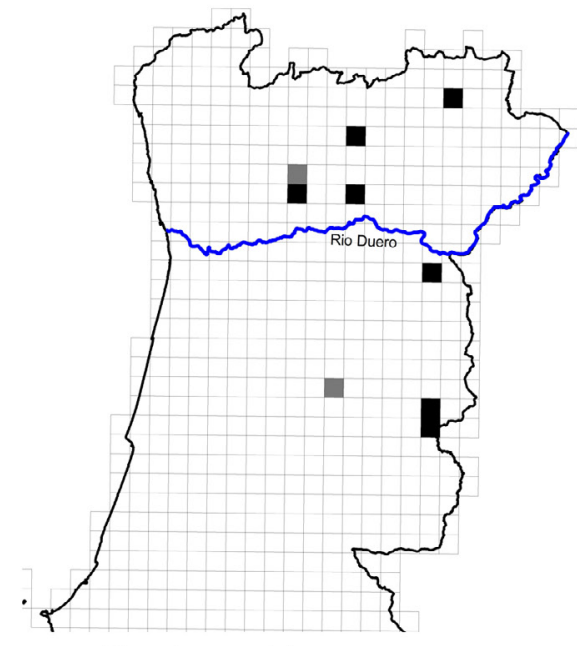

Plecotus austriacus

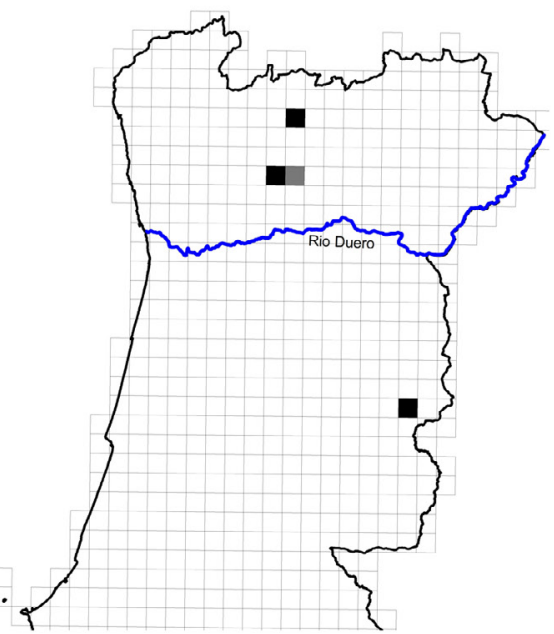

Myotis mystacinus

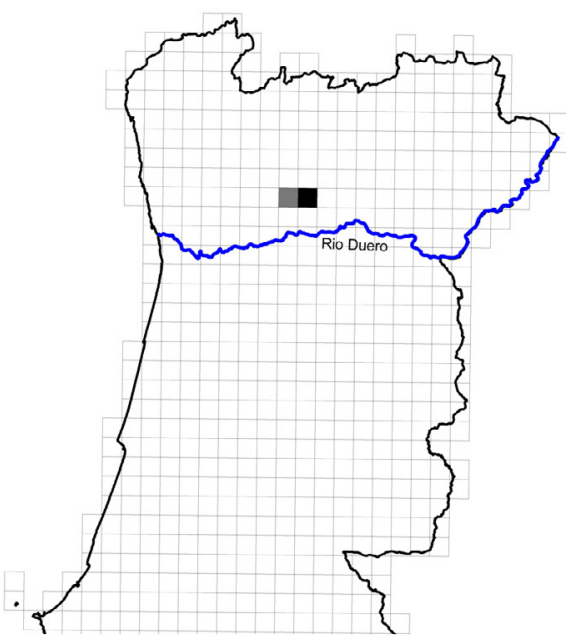

Miniopterus schreibersii

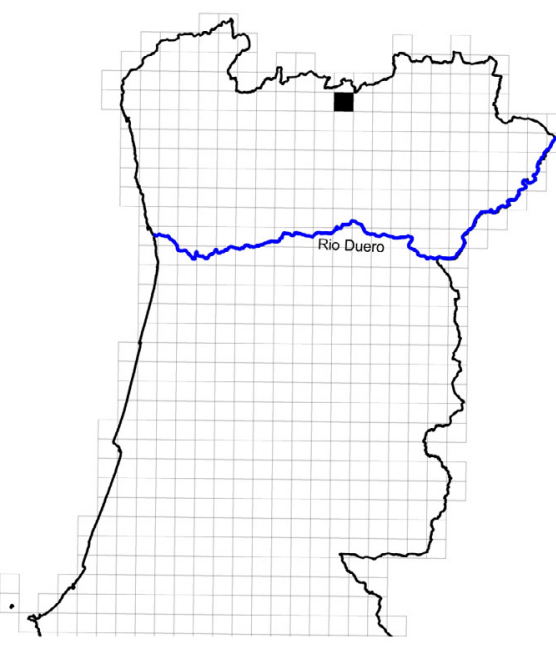

Nyctalus noctula

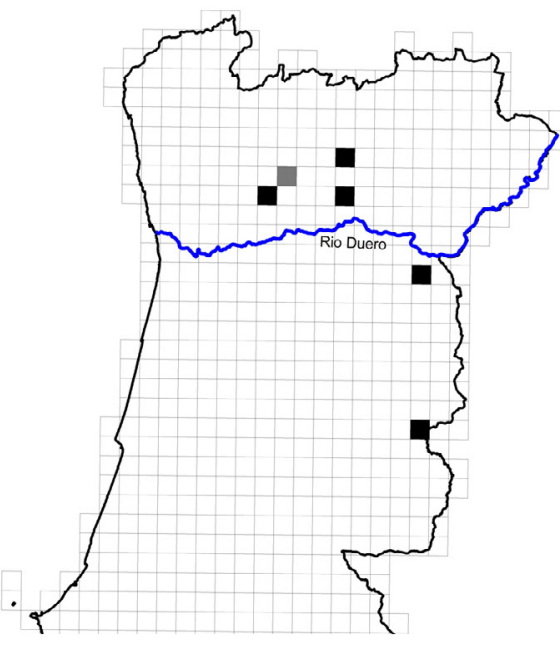

Pipistrellus kulhii

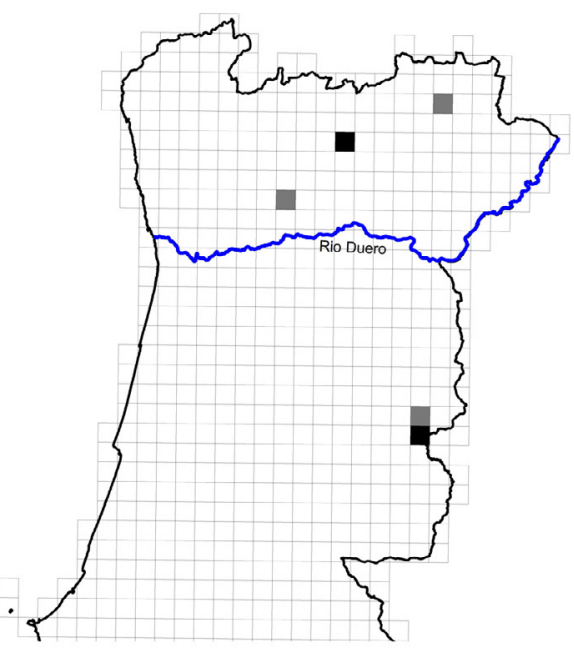

Plecotus auritus

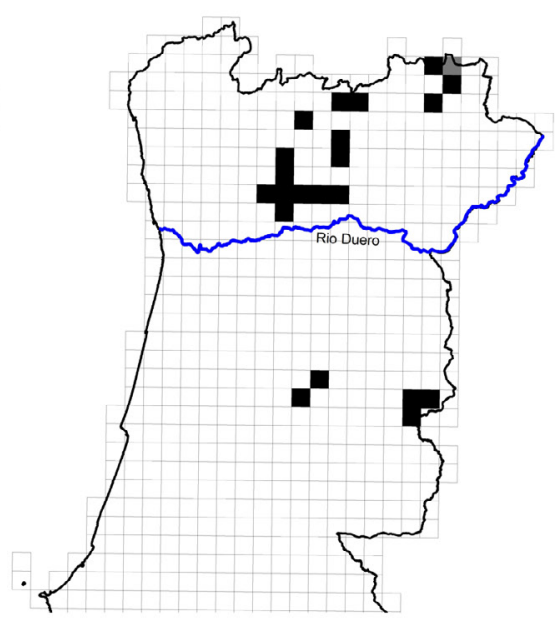

Pipistrelus pipistrellus 


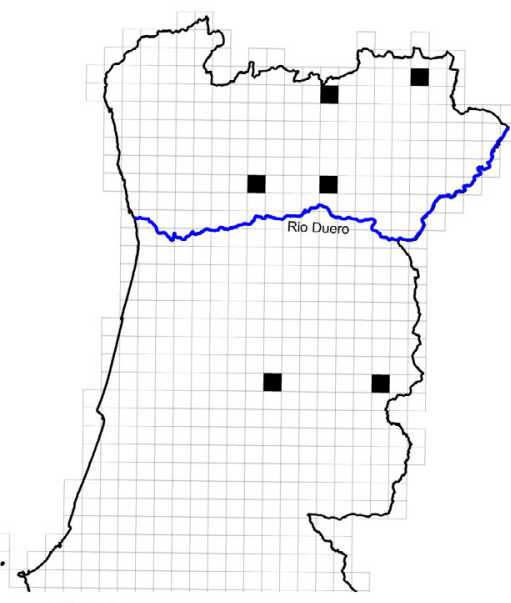

Pipistrellus pygmaeus

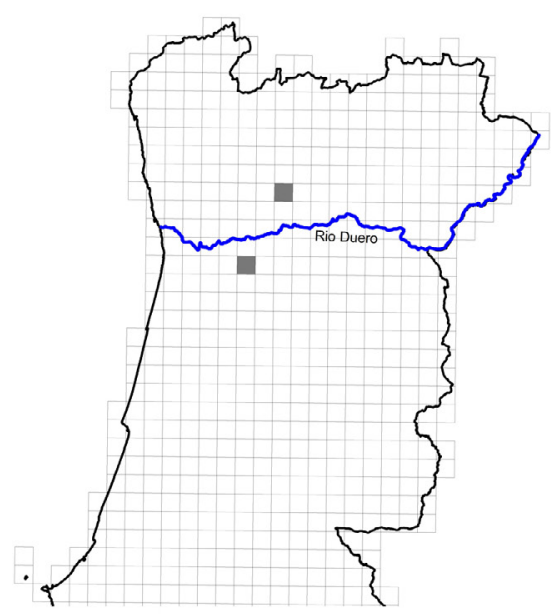

Rhinolophus hipposideros

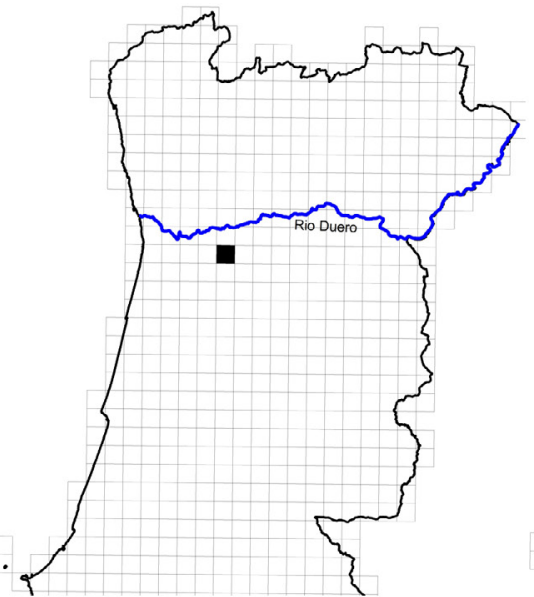

Rhinolophus euryale

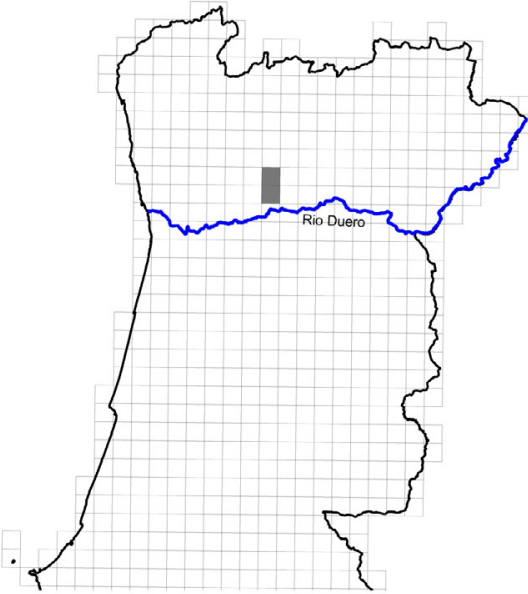

Rhinolophus ferrumequinum 
ANEXo II

Listado de los puntos de muestreo, con el código del lugar, localidad mas próxima, altitud, local de captura, Parque Natural, Lugar de Importancia Comunitaria (LIC), localización a la cuadricula UTM 1X1Km, municipio y hábitat. Las abreviaturas de los Parques y Reservas Naturales son las siguientes: PNA (Parque Natural do Alvão); PNM (Parque Natural de Montesinho); PNSE (Parque Natural da Serra da Estrela) y RNSM (Reserva Natural da Serra da Malcata). Las abreviaturas de los LICs son los siguientes: PTCON0002 (LIC Montesinho /Nogueira), PTCON0003 (LIC Alvão/Marão), PTCON00004 (LIC Malcata) y PTCON0014 (LIC Serra Estrela) y PTCON0014 (LIC Serra Estrela) y PTCON0025 (LIC Serra de Montemuro).

\begin{tabular}{|c|c|c|c|c|c|c|c|c|c|c|c|c|c|c|c|c|c|c|c|c|c|c|c|c|c|c|}
\hline 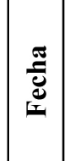 & 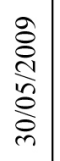 & 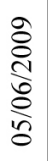 & 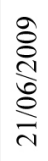 & 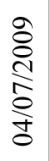 & 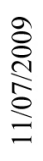 & 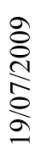 & 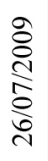 & 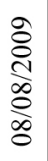 & 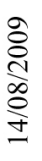 & 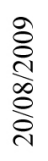 & 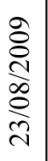 & 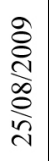 & 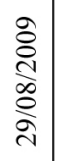 & 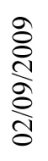 & 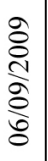 & 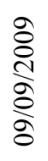 & 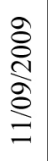 & 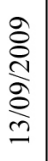 & 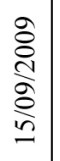 & 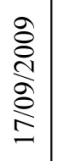 & 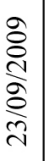 & 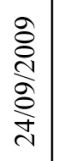 & 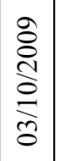 & 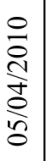 & 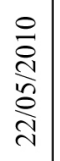 & 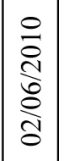 \\
\hline$\tilde{J}$ & $\exists$ & $\mathcal{I}$ & $\mathcal{3}$ & J & 3 & ְּ & $\Xi$ & $\stackrel{\infty}{=}$ & 9 & $\stackrel{\varrho}{=}$ & $\bar{\exists}$ & $\cong$ & $\stackrel{m}{=}$ & $\Xi$ & $\frac{n}{2}$ & $\stackrel{0}{\square}$ & $\Xi$ & $\stackrel{\infty}{\beth}$ & $\frac{\ddots}{\sqsupset}$ & ત્తి & $\vec{\jmath}$ & 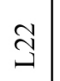 & 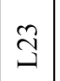 & $\stackrel{\Xi}{\leftrightarrows}$ & $\stackrel{\overbrace{}}{\lrcorner}$ & \\
\hline 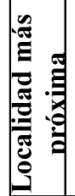 & $\begin{array}{l}0 \\
0 \\
0 \\
0 \\
0 \\
y \\
\text { J } \\
\text { J }\end{array}$ & $\begin{array}{l}\stackrel{\frac{\pi}{5}}{0} \\
\stackrel{0}{0} \\
\frac{0}{2}\end{array}$ & 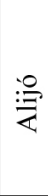 & $\frac{\stackrel{9}{?}}{\gtrless}$ & 을 & $\begin{array}{l}0 \\
0 \\
0 \\
0 \\
0 \\
0 \\
\tilde{G} \\
\text { ज्ञ }\end{array}$ & 芯 & 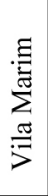 & $\begin{array}{l}\sum_{\frac{1}{\pi}} \\
\frac{\pi}{3} \\
\frac{\pi}{5}\end{array}$ & 号 & $\stackrel{\mathscr{E}}{\Xi}$ & 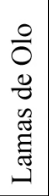 & $\frac{8}{5}$ & $\stackrel{\circ}{\equiv}$ & 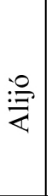 & 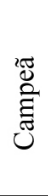 & 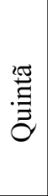 & 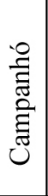 & : & 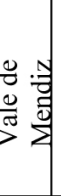 & 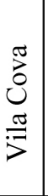 & 离 & 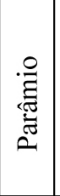 & $\frac{\pi}{0}$ & $\underset{J}{\tilde{U}}$ & \\
\hline
\end{tabular}

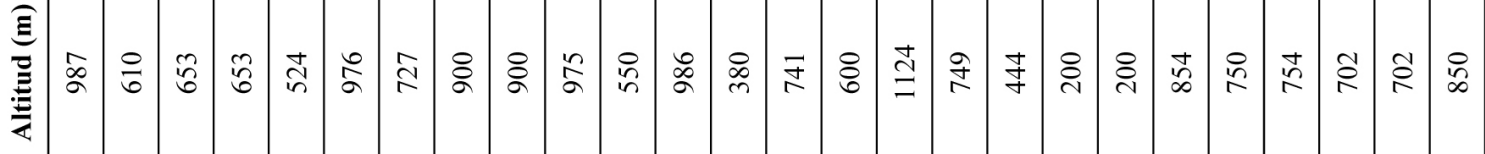

\begin{tabular}{|c|c|c|c|c|c|c|c|c|c|c|c|c|c|c|c|c|c|c|c|c|c|c|c|c|c|c|}
\hline 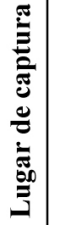 & $\stackrel{0}{2}$ & $\stackrel{\Xi \Xi}{\Xi}$ & 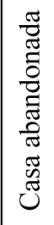 & 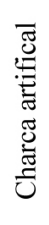 & 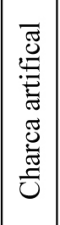 & $\stackrel{\vartheta}{2}$ & $\stackrel{\varrho}{\simeq}$ & $\stackrel{\ominus}{2}$ & 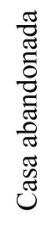 & 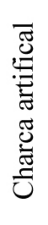 & $\stackrel{0}{2}$ & $\stackrel{\circ}{\approx 2}$ & 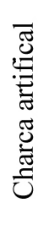 & 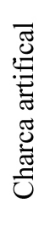 & 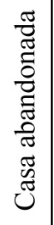 & $\stackrel{\stackrel{\pi}{\Xi}}{\Sigma}$ & $\begin{array}{l}\stackrel{0}{O} \\
\stackrel{E}{*}\end{array}$ & 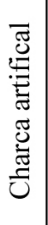 & $\stackrel{\circ}{2}$ & $\stackrel{\circ}{2}$ & $\stackrel{\mathscr{J}}{\Xi}$ & $\stackrel{0}{2}$ & 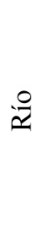 & $\begin{array}{l}0 \\
\frac{0}{\pi} \\
\text { है } \\
\text { 国 }\end{array}$ & 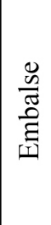 & $\stackrel{2}{\approx}$ \\
\hline
\end{tabular}

\begin{tabular}{|c|c|c|c|c|c|c|c|c|c|c|c|c|c|c|c|c|c|}
\hline 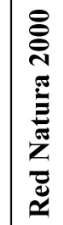 & 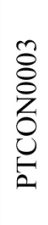 & 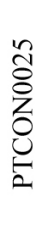 & 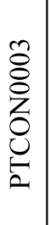 & 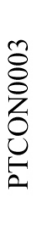 & 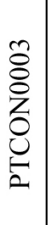 & 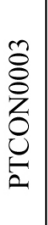 & 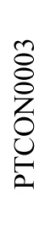 & 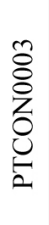 & 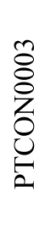 & 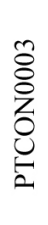 & 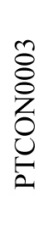 & 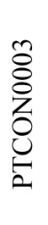 & $\begin{array}{l}0 \\
0 \\
0 \\
0 \\
0 \\
0 \\
\varepsilon\end{array}$ & 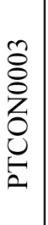 & 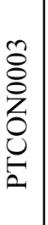 & $\begin{array}{l}\text { ô } \\
8 \\
o \\
0 \\
0 \\
0\end{array}$ & $\begin{array}{l}\text { ¿े } \\
8 \\
0 \\
0 \\
0 \\
0\end{array}$ \\
\hline
\end{tabular}

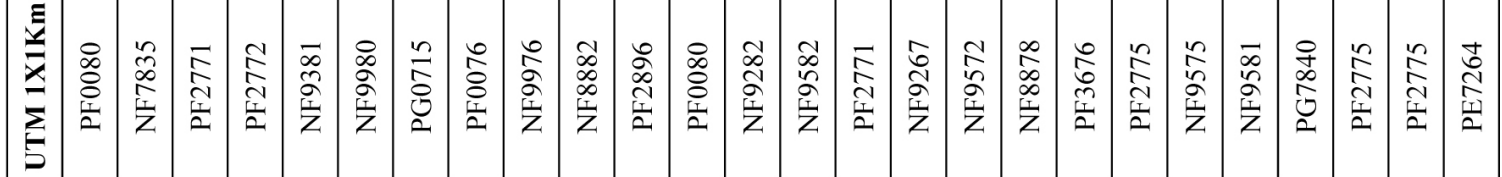

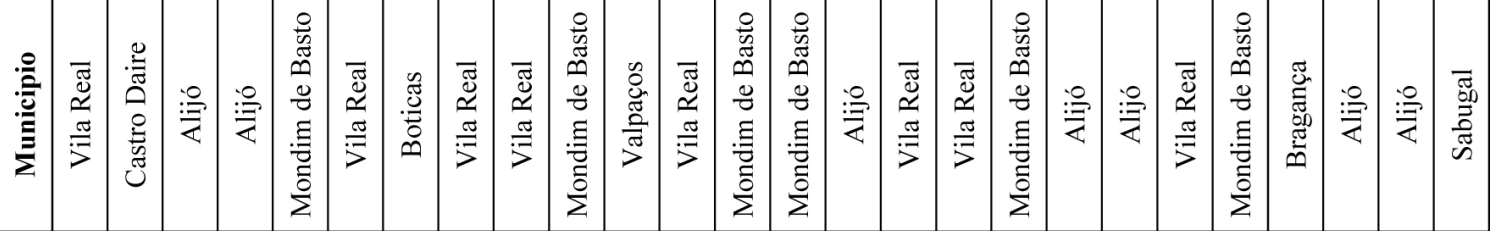

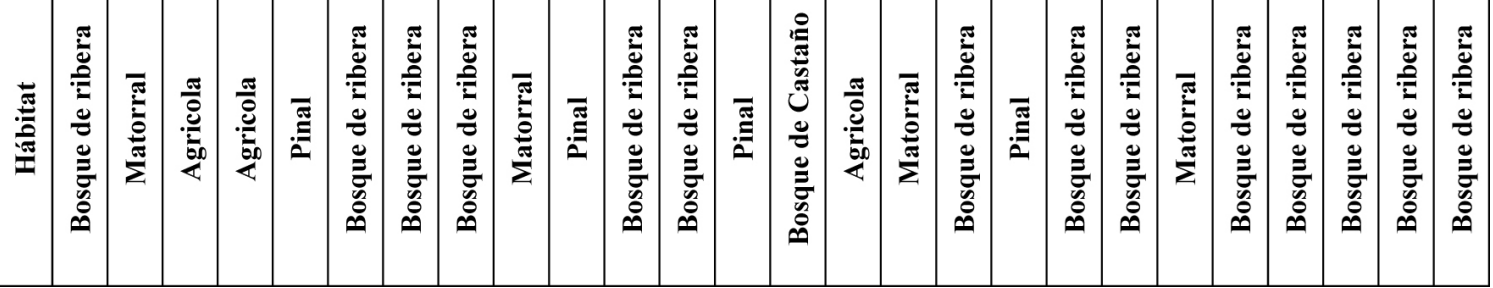




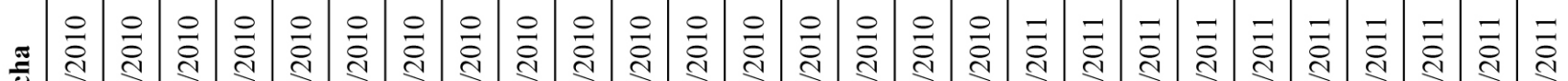

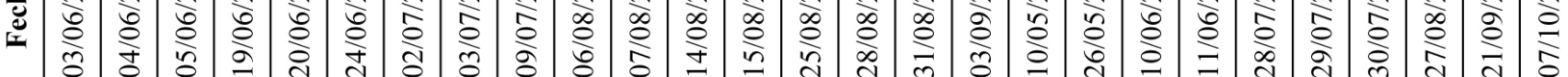

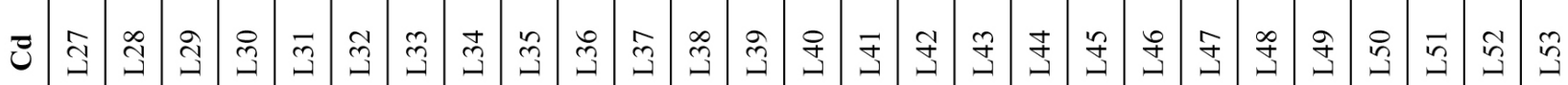

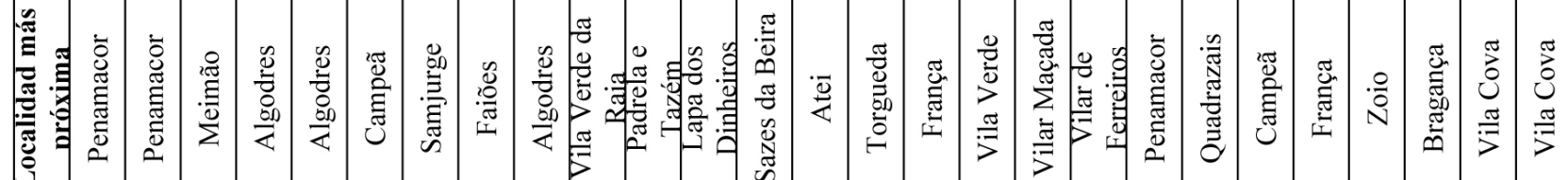

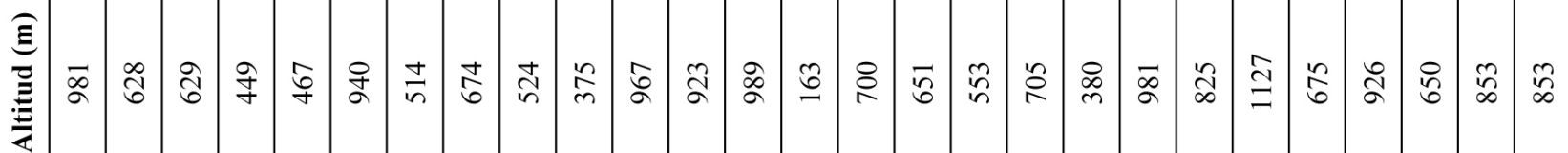

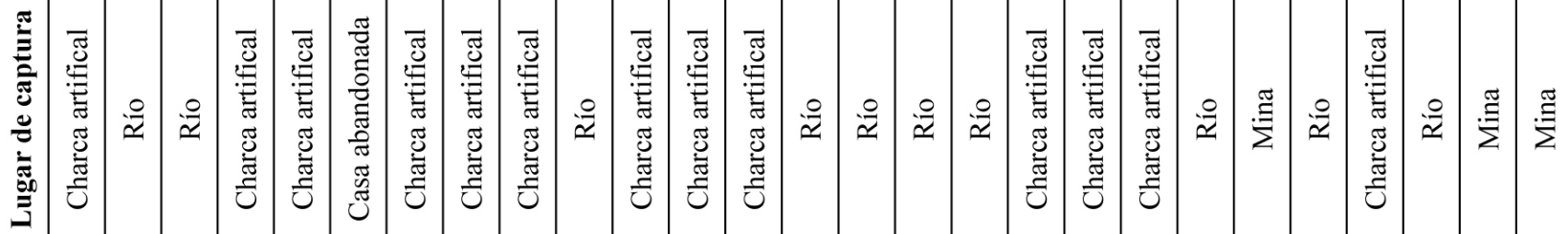

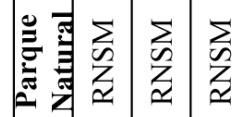

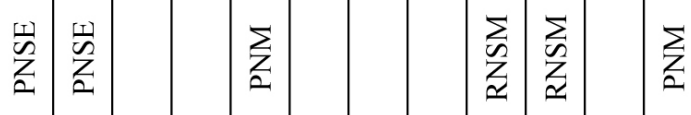

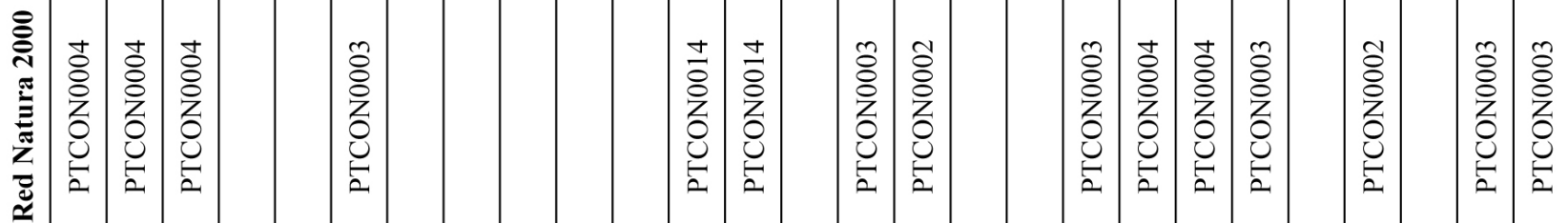

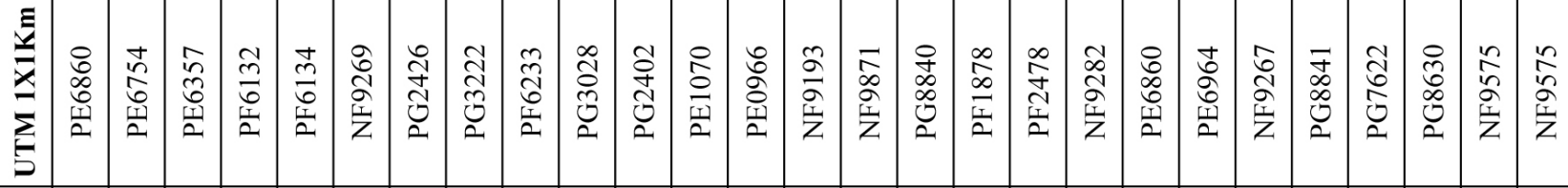

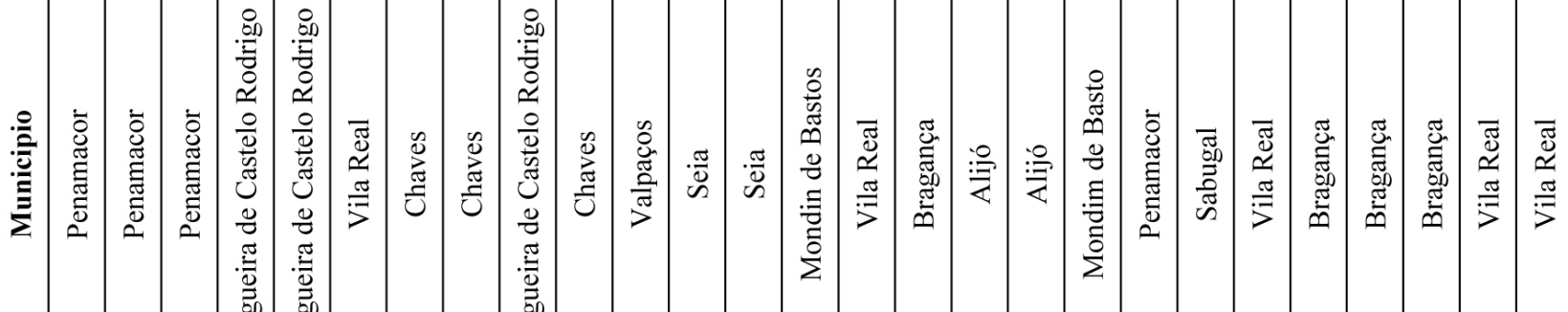

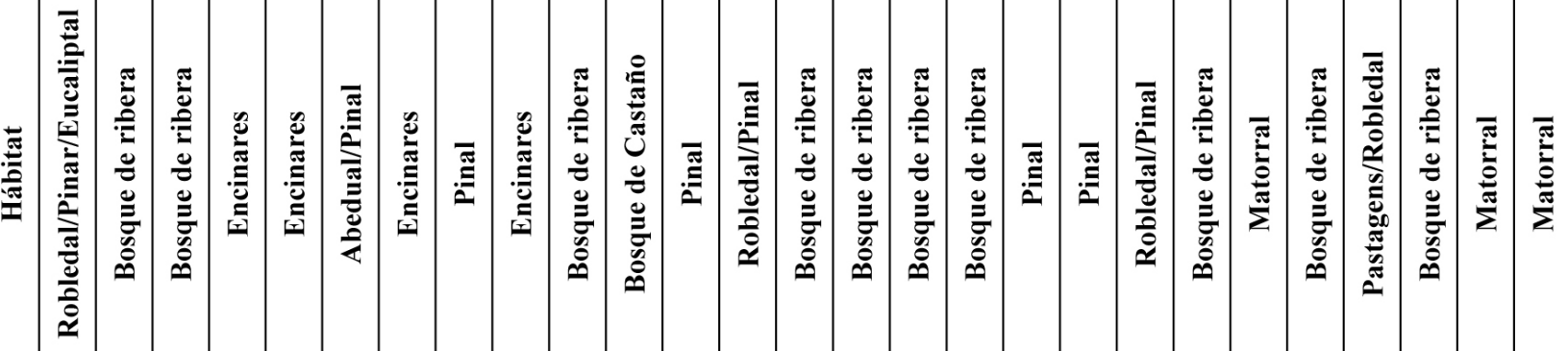

